\title{
Highlights vom Weltkongress für Parkinsonsyndrome in Amsterdam
}

PARKINSON VOM AKINESE-TYP

\section{Achten Sie besonders auf die nicht motorischen Symptome!}

- Nicht motorische Symptome gehören ebenso zum klinischen Bild des Morbus Parkinson wie die charakteristischen Störungen der Beweglichkeit. Diese häufig unterschätzten Krankheitsmerkmale haben überdies einen erheblichen Einfluss auf die Lebensqualität. Besonders genau hinschauen sollte man bei Patienten vom Akinese-Typ, denn sie scheinen überproportional häufig betroffen zu sein. Zu diesem Ergebnis kommt eine im Rahmen eines internationalen Studienprojekts durchgeführte Analyse. Von den 236 parkinsonkranken Teilnehmern wurden $18 \%$ als Tremor-Typ, 30\% als Akinese-/RigorTyp und die restlichen $52 \%$ als Mischtyp klassifiziert. Zur Erfassung der nicht mo- torischen Symptome wurde die 30 Items umfassenden NMSS (Non Motor Symptoms Scale) und zur Exploration der gesundheitsbezogenen Lebensqualität das PDQ-8 (Parkinson's Disease Questionnaire- 8 Items) eingesetzt. Dabei ergaben sich für das „Akinesekollektiv“ bei beiden Messinstrumenten im Mittel signifikant schlechtere Scores als für die Patienten vom Nicht-Akinese-Typ ( $p=0,016$ und $\mathrm{p}=0,045)$. Außer beim Erkrankungsalter (63 versus 59 Jahre) ließen sich weder bei den demografischen, noch bei den klinischen Charakteristika signifikante Unterschiede zwischen den Gruppen erkennen.

Yogini Naidu, London/Großbritannien

\section{MOTORISCHE KOMPLIKATIONEN UNTER LEVODOPATHERAPIE}

\section{Niedriges Körpergewicht begünstigt die Entwicklung von Dyskinesien}

- Bei etwa 40\% der Parkinsonpatienten treten bereits nach vier- bis sechsjähriger Levodopatherapie Dyskinesien auf. Auf der Suche nach intrinsischen und extrinsischen Risikofaktoren hat Dr. Jagdish Sharma, Newark/Großbritannien, die einschlägige Literatur der letzten Jahre durchforstet. Dabei kristallisierten sich als prädiktiv heraus:

- Krankheitsbeginn im frühen Lebensalter,

- lange Krankheitsdauer,

- weibliches Geschlecht,

- genetische Prädisposition.

Zusätzlich zu diesen nicht modifizierbaren Variablen ließ sich aber auch ein Zusammenhang zwischen früher Dyskinesieentwicklung und potenziell beeinflussbaren Faktoren erkennen:

- niedriges Körpergewicht bzw. Gewichtsverlust im Krankheitsverlauf,

- hohe Levodopadosis pro kg Körpergewicht (prädiktiver als die absolute Höhe der Levodopadosis).

Nach Sharmas Interpretation ergibt sich daraus ein logischer Zusammenhang - je weniger ein Parkinsonpatient wiegt, desto höher ist seine Levodopadosis pro kg Körpergewicht. Das erklärt - zumindest zum Teil - auch das bei Frauen überproportional höhere Dyskinesierisiko. Wegen ihres in der Regel niedrigeren Körpergewichts sind sie per se einer stärkeren Levodopaexposition ausgesetzt.

Jagdish Sharma, Newark/Großbritannien

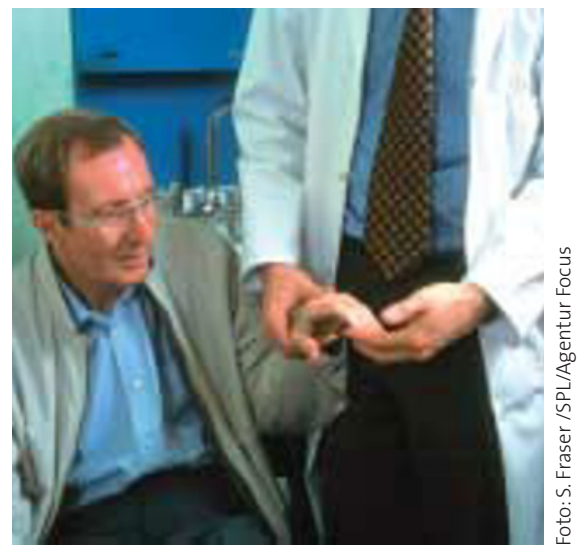

Wie hoch ist sein Dyskinesierisiko?

\section{KOGNITIVE DYSFUNKTION}

\section{Erhöht Hyperhomozystein- ämie das Demenzrisiko?}

- Hohe Homozysteinspiegel wirken sich bei Parkinsonpatienten ungünstig auf die kognitive Leistungsfähigkeit aus und könnten ein Risikofaktor für die Entwicklung einer Demenz sein. Zu diesem Schluss kommt eine japanische Arbeitsgruppe nach dem Vergleich der Werte von Betroffenen ohne und mit kognitiver Dysfunktion - evaluiert per MMSE (Mini Mental State Examination), WAIS-R (Wechsler Adult Intelligence Scale-Revised) und SDS (Self-Rating Depression Scale) - sowie von Patienten mit Alzheimerdemenz. Das Plasmahomozystein korrelierte positiv mit dem Alter der Probanden und negativ mit den ebenfalls gemessenen VitaminB12- und Folsäurekonzentrationen. In der Gruppe der kognitiv gestörten Patienten zeigte sich zudem eine inverse Wechselbeziehung mit dem IQ-Score der WAIS-R. Hisanori Kowa, Yonago/Japan

\section{PARKINSONPATIENTEN MIT MIGRÄNE}

\section{Kopfschmerzattacke nach Unterbrechung der Therapie}

— Es wird diskutiert, dass Störungen im Dopaminsystem auch in der Pathogenese der Migräne eine Rolle spielen könnten. Dazu steuerte eine griechische Arbeitsgruppe eine interessante Beobachtung bei. Das von ihnen untersuchte Kollektiv umfasste zwölf Parkinsonkranke mit Migräneanamnese. Bei sieben Patienten löste das nebenwirkungsbedingte temporäre oder dauerhafte Absetzen der dopaminergen Therapie einen Migräneanfall aus. Die in allen Fällen vor und nach Abbruch der Levodopa-/Dopaminagonisten-Therapie durchgeführte 24-Stunden-EEG zeigte bei den Betroffenen und zwei Patienten ohne Kopfschmerzen das für eine Migräneattacke typische Entladungsmuster.

Dimitrios Kountouris, Athen/Griechenland BL-KI -

- Weltkongress für Parkinsonsyndrome in Amsterdam/Niederlande, XVII WFN World Congress on Parkinson's Disease and Related Disorders, 9.-13.12. 2007 in Amsterdam/Niederlande 